Article

\title{
Common-Mode Noise Reduction in Noncontact Biopotential Acquisition Circuit Based on Imbalance Cancellation of Electrode-Body Impedance
}

\author{
Minghui Chen ${ }^{1} \mathbb{D}$, Jianqing Wang ${ }^{1, *}$, Daisuke Anzai ${ }^{1}$, Georg Fischer ${ }^{2} \mathbb{C}$ and Jens Kirchner ${ }^{2} \mathbb{C}$ \\ 1 Department of Electrical and Mechanical Engineering, Nagoya Institute of Technology, Gokiso-cho, \\ Showa-ku, Nagoya 466-8555, Japan; m.chen.032@stn.nitech.ac.jp (M.C.); Anzai@nitech.ac.jp (D.A.) \\ 2 Institute for Electronics Engineering, Friedrich-Alexander-Universität of Erlangen-Nuremberg, \\ Schlossplatz 4, 91054 Erlangen, Germany; georg.fischer@fau.de (G.F.); jens.kirchner@fau.de (J.K.) \\ * Correspondence: wang@nitech.ac.jp
}

Received: 9 November 2020; Accepted: 10 December 2020; Published: 13 December 2020

\begin{abstract}
Biopotential sensing technology with electrodes has a great future in medical treatment and human-machine interface, whereas comfort and longevity are two significant problems during usage. Noncontact electrode is a promising alternative to achieve more comfortable and long term biopotential signal recordings than contact electrode. However, it could pick up a significantly higher level of common-mode (CM) noise, which is hardly solved with passive filtering. The impedance imbalance at the electrode-body interface is a limiting factor of this problem, which reduces the common mode rejection ratio (CMRR) of the amplifier. In this work, we firstly present two novel CM noise reduction circuit designs. The circuit designs are based on electrode-body impedance imbalance cancellation. We perform circuit analysis and circuit simulations to explain the principles of the two circuits, both of which showed effectiveness in CM noise rejection. Secondly, we proposed a practical approach to detect and monitor the electrode-body impedance imbalance change. Compared with the conventional approach, it has certain advantages in interference immunity, and good linearity for capacitance. Lastly, we show experimental evaluation results on one of the designs we proposed. The results indicated the validity and feasibility of the approach.
\end{abstract}

Keywords: biomedical electronics; common-mode noise; electromagnetic interference; noncontact electrode

\section{Introduction}

As the aging society problem draws great attention, the sensing technology of biosignals, especially biopotential signals like electrocardiogram (ECG), electroencephalogram (EEG) or electrooculogram (EOG), has advanced tremendously over the years. As an example, the body area network (BAN) with wearable sensing technologies [1-3] collects vital data for health-state monitoring, which is considered as an emerging solution to soaring healthcare costs and shortages of medical resources.

In addition to medical treatment and healthcare, the biosignals could also be considered as a significant interface between human body and machines. In the literature, applications like activity recognition [4], driving assistance [5,6] or human-computer interface [7-9] were mentioned by researchers. The potential of biosignals is still to be exploited when sensing technologies advance further. Comfort, longevity and precision are three main topics about the advancements of electrode-based sensing technology.

Wet electrode is the most common type for both clinical and research applications. A typical wet electrode consists of a silver-silver chloride metal that is surrounded by a wet or solid hydrogel, containing chloride. The primary drawbacks with wet electrodes are limited lifetime, discomfort 
and skin irritations. Wet electrodes degrade as the moisture content evaporates, limiting its useful lifetime to several hours, or at most a few days. The oxidation processes may also have some influence, but normally not mentioned as the main factor. Dry contact electrodes operate without the use of wet/gel coupling media. The metal in the electrode directly contacts the skin to couple biopotential signals. However, dry contact electrodes still rely on some degree of moisture. The performance of a dry contact electrode usually increases over time as more moisture permeates the skin-electrode interface.

The final type of electrodes, noncontact, can be thought of as a special case of dry electrodes. The noncontact electrode was introduced by Lopez and Richardson in 1967 [10] and further developed in the last decade [11-14]. Noncontact electrodes have certain advantages in biosignal acquisition because they do not need direct skin contact, and they can be integrated in objects like beds or cars for long-term biosignal recording. They operate not only without gel, but also through an insulation layer such as clothing. Therefore, they are also called capacitive electrodes. There are also some other technologies that can achieve noncontact sensing of biosignals, like using the Doppler cardiogram [15] or a radar [16-19]. Recently, Taylor et al. [20] provided a noncontact approach to detect COVID-19.

The coupling capacitance of a noncontact electrode can range in the order of tens or hundreds of picofarad [21]. Obtaining acceptable signals requires the use of high input impedance active electrodes. Impedance at the electrode-body interface (EBI) of noncontact electrodes are highly sensitive to environmental conditions such as humidity and the exact insulating material. This property makes the noncontact electrodes induce much more noise than wet electrodes, because the imbalance of the EBI impedances allows the common-mode (CM) noise converting into a differential mode (DM) interference voltage [22]. Liao et al. [23] explained to what extent an imbalance in the impedances of EBI could reduce the common mode rejection ratio (CMRR). As a result, CM noise source like the power line, electromagnetic interference (EMI) or a wireless power transfer system [23] can severely interfere with the detection progress. Moreover, because of this, a body or skin motion could change the coupling conditions such that the imbalance varies and induces more noise; cancelling the imbalance can also reduce motion artefacts thanks to improving balance, thus raising CMRR.

There are also imbalances in other circuit elements. Perfect circuit elements for complete impedance matching in sensing circuits is not a practical issue in the industry. Because the biopotentials are generally at very low voltage level (microvolts or millivolts) and almost the same frequency ( 0 to $1 \mathrm{kHz}$ ) as the noise, a small deviation of impedance value may lead to a noise at millivolts in worst case. The well-known instrumentation amplifier (INA) uses two amplifying buffers and a bridging resistor to restore the CMRR of a differential amplifier, which is a common strategy for biopotential acquisition nowadays. However, for the case of noncontact electrodes, it is not possible to acquire high quality biosignals only by using an INA, especially when there is a body or skin motion. Chi et al. [24] expressed the CMRR in the following shape:

$$
C M R R \approx \frac{Z_{i}}{\left|Z_{1}-Z_{2}\right|}
$$

where $Z_{1}$ and $Z_{2}$ are the EBI impedances, $Z_{i}$ is the input impedance of the biopotential amplifier. At low frequencies like $50 / 60 \mathrm{~Hz}$, the impedances of noncontact EBIs are primarily capacitive and the expression simplifies to

$$
C M R R \approx \frac{C_{1} C_{2}}{C_{i}\left|C_{1}-C_{2}\right|}
$$

From (2), we can see that for noncontact/capacitive electrodes, an imbalance of picofarads between the electrodes can reduce the CMRR more severely than contact ones, whose imbalance is usually in the order of kiloohms. This fact is the reason why a capacitive sensing scheme is so sensitive to imbalance variations.

In our previous work [25], we presented the principle of a CM noise reduction approach for contact biopotential acquisition. Our work on contact electrodes basically shares the same principles with this work in cancelling the imbalance of EBI. A pair of digital potentiometers (DigiPOTs) were employed 
to compensate the imbalance of EBI resistance. DigiPOTs has a certain advantage in cancelling the imbalance of resistance in the EBI of contact electrodes, because they can be tuned exactly and conveniently with a digital control signal. However, when it comes to noncontact electrodes, it will not work as successfully as for the contact electrodes, because the imbalance becomes capacitive. Serteyn et al. [26] proposed an approach to exploit an injection signal to track the capacitance change of EBI and restore the ECG signal through digital processing. Their approach taught us how to monitor the imbalance change at the EBI, but their work did not solve the imbalances caused by the other circuit elements.

In this work, we propose using digitally tunable capacitor (DTC) to cancel the imbalance of EBI capacitance. A DTC is an IC variable capacitor that can be controlled with a digital signal. We also use an injection signal to monitor the variance of EBI capacitance, whereas the circuit design is completely original with a microcomputer to control the DTC and reject the CM noise, which could also work on the imbalance of the other circuit elements, as we mentioned. As in [25], we use a pseudo EMI noise source in simulation evaluation to quantitatively show the effectiveness of our approach. We also use circuit boards to confirm its validity and feasibility in a laboratory environment.

\section{Circuit Analysis}

\subsection{Proposal 1: "DTC Series"}

In this section, we present our biopotential acquisition circuit design proposals and analyze how they work in reducing the CM noise. The assumed acquisition model and the conditions of our circuit analysis are shown in Figure 1. There are two noncontact sensing electrodes and one ground electrode. The biopotential signals are acquired from the two sensing electrodes. The EBI capacitance is denoted as $C_{e a}$ or $C_{e b}$. Usually, they are unbalanced due to the different attachment conditions. $C_{e g}$ denotes the impedance of the ground electrode. In addition, the stray capacity between the circuit ground and the earth ground is denoted as $C_{s}$. The interference voltage $V_{C M}$ is between the human body and the earth ground. This model could be considered as a usual one for single channel ECG, EEG, EOG, etc. Theoretically, a 2-wired sensing circuit can also be employed to acquire one channel of biopotential, but a ground electrode or a driven-right-leg (DRL) electrode is generally necessary to reject noise and reduce errors.

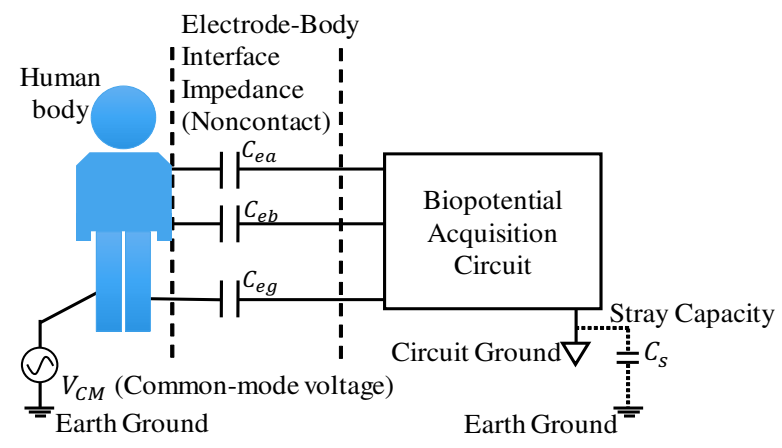

Figure 1. Assumed acquisition model and conditions of circuit analysis.

Figure 2 shows the CM equivalent circuit of our first proposal, which we call "DTC Series". It is based on the noncontact 3-wired single channel biopotential acquisition model shown in Figure 1. Two buffer amplifiers are used to provide high input impedance. There are two grounds for the circuits in Figure 2 and the other circuits we handle. One is the circuit ground for differential mode voltage. The operational amplifier (OP-AMP)'s ground is this ground and the OP-AMP circuit thus works normally. The other ground is the earth ground for common mode voltage. This ground is floating, and the floating is difficult to be solved by autozero or a similar mechanism because it is the earth. The input to the OP-AMP contains both the differential mode voltage and the common mode voltage, 
and the common mode voltage is converted into a differential voltage as a noise at the OP-AMP output. Between the electrodes and the buffers, two DTC elements, $C_{t 1}$ and $C_{t 2}$, are installed in series to the input impedance of the buffer. The biopotential signals are differentially amplified with an OP-AMP. The OP-AMPs are not ideal because if they were, the currents $i_{a}$ and $i_{b}$ in Figure 2 would become zero and the CM noise would not affect this circuit. The input offset voltages of the buffers are designed to be very low, such that the input and output voltages of them are assumed to be equal. With the conditions that we gave, $V_{\text {out } 1}$ can be written as

$$
V_{\text {out } 1}=\frac{j \omega C Z_{\text {eg }} R_{1}\left(R_{1}+R_{2}\right)\left(Z_{\text {eg }}+Z_{\text {in }}\right)\left(Z_{a}-Z_{b}\right) V_{C M}}{R_{2}\left(Z_{\text {ea }}+Z_{\text {in }}\right)\left[\left(1+j \omega C Z_{\text {eg }}\right)\left(R_{1}+R_{2}\right)\left(Z_{\text {eg }}+Z_{\text {in }}\right)+Z_{e g}\left(Z_{a}-Z_{b}+Z_{\text {in }}\right)\right]}
$$

where

$$
Z_{a}-Z_{b}=\left(\frac{C_{e a}-C_{e b}}{j \omega C_{e a} C_{e b}}+\frac{C_{t 1}-C_{t 2}}{j \omega C_{t 1} C_{t 2}}\right)
$$

$Z_{\text {in }}$ is the input impedance (with respect to the circuit ground) of the amplifier U1 or U2 (buffers). From (3), we can know that if $\left(Z_{a}-Z_{b}\right)$ equals zero, then the output of CM noise $V_{\text {out }}$ also becomes zero. Therefore, we can adjust the capacitance of $C_{t 1}$ and $C_{t 2}$ to cancel the imbalance between $C_{e a}$ and $C_{e b}$, such that the $\mathrm{CM}$ noise is reduced. Besides, this imbalance cancellation is independent of the frequency, which means that theoretically we can drive down the $\mathrm{CM}$ noise of all frequencies to extremely low level once $C_{t 1}$ and $C_{t 2}$ are exactly adjusted.

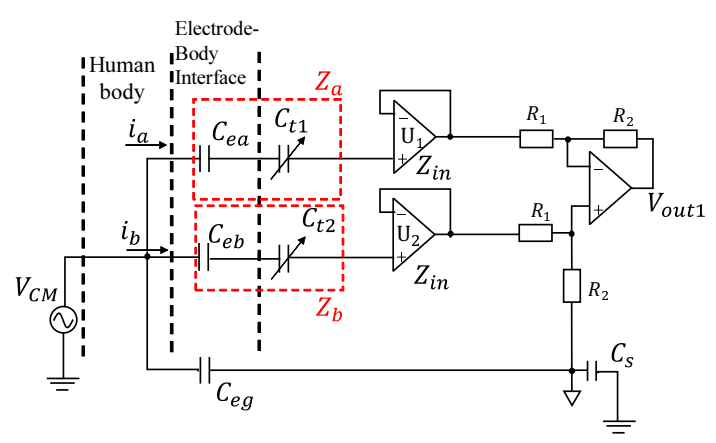

Figure 2. Common-mode (CM) equivalent circuit of digitally tunable capacitor series ("DTC Series").

\subsection{Proposal 2: "DTC Bypath"}

There is another circuit design to achieve capacitive imbalance cancellation. Its $\mathrm{CM}$ equivalent circuit is shown in Figure 3. This design tries to make the voltage at $V_{a}$ and $V_{b}$ equal, like the famous Wheatstone bridge. We call this proposal the "DTC Bypath". In this circuit, $V_{\text {out } 2}$ can be written as

$$
V_{\text {out } 2}=\Psi \cdot \frac{\Gamma}{\Pi} \cdot V_{C M}
$$

where

$$
\begin{gathered}
\Gamma=\omega^{2} Z_{i n}\left(C_{e a} C_{t 2}-C_{e b} C_{t 1}\right)+j \omega\left(C_{e b}-C_{e a}\right) \\
\Psi=\frac{j \omega C_{s}\left(R_{1}+R_{2}\right)\left[j \omega\left(C_{e b}+C_{t 2}\right) Z_{i n}+1\right]}{R_{2} j \omega\left(C_{e b} Z_{i n}+j \omega\left(C_{e g}+C_{s}\right)\left[j \omega\left(C_{e b}+C_{t 2}\right) Z_{i n}+1\right]\left(R_{1}+R_{2}\right)\right.} \\
\Pi=-\omega^{2} Z_{i n}^{2}\left(C_{e b}+C_{t 2}\right)\left(C_{e a}+C_{t 1}\right)+j \omega\left(C_{e a}+C_{t 1}+C_{e b}+C_{t 2}\right)
\end{gathered}
$$

$\Psi$ and $\Pi$ are coefficients given by (7) and (8). If we want $V_{\text {out2 }}$ to be zero, which can be derived by letting $\Gamma=0$, we can obtain

$$
\frac{C_{e a}}{C_{e b}}=\frac{j \omega C_{t 1} Z_{i n}+1}{j \omega C_{t 2} Z_{\text {in }}+1}
$$


This equation can also be derived by letting $V_{a}=V_{b}$, where $V_{a}$ and $V_{b}$ are the input voltages of the buffers denoted in Figure 3. From this equation, we can see that the CM noise can be reduced by adjusting the $C_{t 1}$ and $C_{t 2}$. It should be noted that (9) is affected by frequency, and if $\omega Z_{i n}$ is large enough, (9) becomes

$$
\frac{C_{e a}}{C_{e b}}=\frac{C_{t 1}}{C_{t 2}}
$$

It reveals that by employing "DTC Bypath", we can reduce the CM noise through matching $C_{e a} / C_{e b}$ and $C_{t 1} / C_{t 2}$.

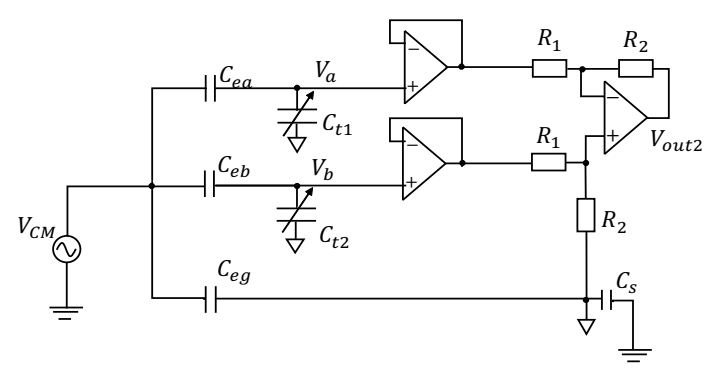

Figure 3. CM equivalent circuit of "DTC Bypath".

\section{Circuit Simulation and Results}

\subsection{Without the DTCS}

In this section, we use the SPICE (simulation program with integrated circuit emphasis) to show simulation results of our proposal, in comparison with a circuit without the DTCs.

Figure 4 shows the CM equivalent circuit for biopotential acquisition without the DTCs. Parameters of the simulated circuit components are shown in Table 1. All the OP-AMPs in the circuit are simulated with the model of OP07 (analog devices), because it has very low input offset voltage, low input bias current and high open-loop gain, which make it suitable for high gain instrumental applications.

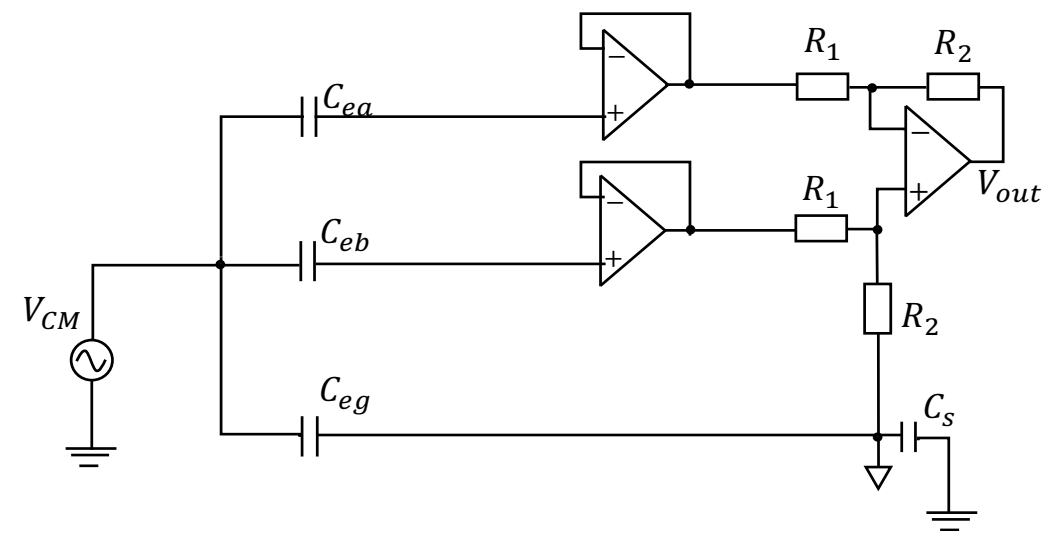

Figure 4. CM equivalent circuit without the DTCs.

Table 1. Parameters of circuit components in Figure 4.

\begin{tabular}{cccc}
\hline Parameter & Value & Parameter & Value \\
\hline Frequency & $60 \mathrm{~Hz}$ & $V_{C M}$ & $1 \mathrm{~V}$ \\
$C_{e a}$ & $1 \mathrm{pF}$ to $400 \mathrm{pF}$ & $R_{1}$ & $10 \mathrm{k} \Omega$ \\
$C_{e b}$ & $200 \mathrm{pF}$ & $R_{2}$ & $10 \mathrm{M} \Omega$ \\
$C_{e g}$ & $200 \mathrm{pF}$ & $C_{s}$ & $200 \mathrm{pF}$ \\
\hline
\end{tabular}


Figure 5 shows the simulated result of output voltage normalized to $\mathrm{CM}$ noise for capacitance imbalance $(-200 \mathrm{pF}$ to $200 \mathrm{pF})$, i.e., the value of $\left(C_{e a}-C_{e b}\right)$ in Figure 4 . When using a noncontact circular electrode, the capacitance can be calculated from

$$
C=\varepsilon_{r} \varepsilon_{0} \frac{\pi r^{2}}{d}
$$

Suppose that there is a stiff plate ECG noncontact electrode with a radius $r$ of $2 \mathrm{~cm}$, and the relative permittivity $\varepsilon_{r}$ of clothes is about 5 to 10 . The thickness $d$ between the human body and the electrode could be about 0.1 to $0.5 \mathrm{~mm}$, depending on the clothes. With these parameters, the EBI capacitance could be 100 to $200 \mathrm{pF}$. Therefore, we assume that the capacitance of $C_{e a}$ and $C_{e b}$ are in hundred picofarad order. Ha et al. [21] also mentioned the approximate value of noncontact electrodes' coupling capacitance as $1 \mathrm{pF}$ to $10 \mathrm{nF}$, depending on their type and attachment conditions. From Figure 5, it can be seen that when the absolute value of imbalance increases, the noise output becomes larger. If $C_{e a}$ is near to 0 (which means that the attachment to human body is near to open), the $\mathrm{CM}$ noise increases severely. If the capacitances of $C_{e a}$ and $C_{e b}$ are matched exactly, $V_{o u t} / V_{C M}$ becomes $-150 \mathrm{~dB}$, which implies that the noise output is near to zero when there is no imbalance. Generally, the imbalance of noncontact EBI capacitance is tens of picofarads or larger [27].

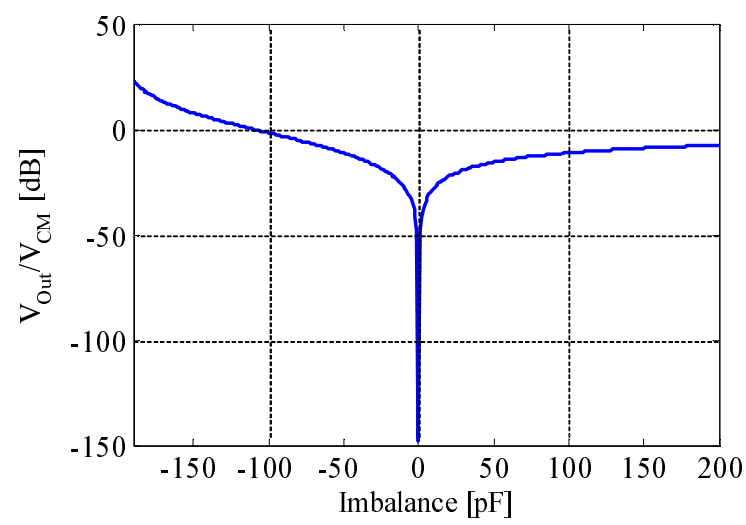

Figure 5. Simulated result of output voltage normalized to CM noise input for capacitance imbalance $-200 \mathrm{pF}$ to $200 \mathrm{pF}$ (Table 1's condition).

\section{2. $V_{\text {out }} / V_{C M}$ vs. $C M R R$}

$V_{\text {out }} / V_{C M}$ is a simple parameter we use in this work to evaluate how much noise could be converted from an external interference $C M$ voltage. $V_{\text {out }}$ is with respect to the circuit ground and $V_{C M}$ is with respect to the earth ground. It can be converted to the CMRR by using the following equations:

$$
C M R R=20 \log \left(\frac{A_{D M}}{A_{C M}}\right)
$$

In our simulation conditions, the effective $\mathrm{CM}$ voltage (with respect to the circuit ground) $V_{\mathrm{CMeff}}$ and $V_{C M}$ has the following relation (when $V_{C M}$ is $1 \mathrm{~V}$ at $60 \mathrm{~Hz}$ ):

$$
20 \log \left(\frac{V_{C M e f f}}{V_{C M}}\right) \approx-6 \mathrm{~dB}
$$

Moreover, the common mode gain $A_{C M}$ in CMRR can be written as:

$$
A_{C M}=\frac{V_{\text {out }}}{V_{C M e f f}}
$$


Differential mode gain $A_{D M}$ is normally a fixed value $60 \mathrm{~dB}$, which almost does not change in the simulation due to the high input impedance. Thus, we can obtain

$$
C M R R \approx 54 \mathrm{~dB}-20 \log \frac{V_{\text {out }}}{V_{C M}}
$$

For example, when $V_{\text {out }} / V_{C M}$ is $-150 \mathrm{~dB}, \mathrm{CMRR}$ is about $204 \mathrm{~dB}$. Generally, this value will be about $100 \mathrm{~dB}$ for an actual instrumentation amplifier at $60 \mathrm{~Hz}$, because the error in the circuit elements could reduce the CMRR by about 40 to $60 \mathrm{~dB}$. We use $V_{\text {out }} / V_{C M}$ as the main benchmark, because high total CMRR is not the main aim in this work.

\section{3. "DTC Series"}

Next, we perform another simulation to confirm the effectiveness of our proposal "DTC Series". Table 2 shows the parameters we used to simulate the circuit elements in Figure 2. The other parameters are the same as we have shown in Table 1. Figure 6 shows the simulated result of output voltage normalized to CM noise for "DTC Series". $C_{t 1}$ is tuned from $300 \mathrm{pF}$ to $500 \mathrm{pF}$, and we can see that $V_{\text {out }} / V_{C M}$ becomes $-150 \mathrm{~dB}$ when $C_{t 1}$ is $400 \mathrm{pF}$. At this point, capacitances of $C_{t 1}$ and $C_{t 2}$ compensate the imbalance between $C_{e a}$ and $C_{e b}$ exactly, such that the $C M$ noise is extremely reduced. Comparing this result with Figure 5, this circuit can drive down the $V_{C M}$ by more than $20 \mathrm{~dB}$ if the imbalance is cancelled to below $20 \mathrm{pF}$.

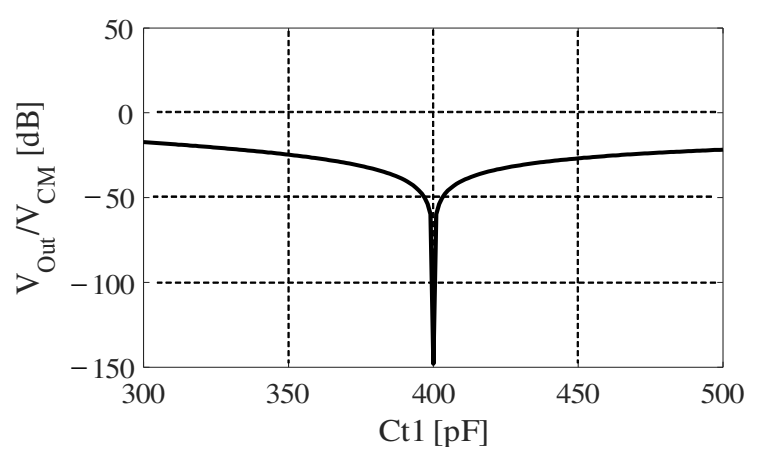

Figure 6. Simulated result of output voltage normalized to CM noise for "DTC Series" under Table 2's condition.

Table 2. Parameters of the simulated circuit components in Figure 2.

\begin{tabular}{cccc}
\hline Parameter & Value & Parameter & Value \\
\hline Frequency & $60 \mathrm{~Hz}$ & $V_{C M}$ & $1 \mathrm{~V}$ \\
$C_{e a}$ & $200 \mathrm{pF}$ & $C_{t 1}$ & $300 \mathrm{pF}$ to $500 \mathrm{pF}$ \\
$C_{e b}$ & $400 \mathrm{pF}$ & $C_{t 2}$ & $200 \mathrm{pF}$ \\
\hline
\end{tabular}

\section{4. "DTC Bypath"}

We performed a simulation in almost the same way on "DTC Bypath". The parameters of the circuit components in this simulation are shown in Table 3, and the results are shown in Figure 7. Because $C_{t 2}$ is $400 \mathrm{pF}$, by calculation we can know that $C_{t 1}$ should be about $199.5 \mathrm{pF}$ to meet the relation in (9), which matches the simulated result in Figure 7. At its lowest point, $V_{\text {out }} / V_{C M}$ becomes about $-70 \mathrm{~dB}$. By comparing the two results in Figures 6 and 7, we can see that generally "DTC Series" works better than "DTC Bypath" in the same conditions. This is because "DTC Bypath" did not completely cancel the imbalance like "DTC Series" in this simulation. According to (9), if we want to completely cancel the imbalance, $C_{t 1}$ and $C_{t 2}$ should be two very large values. However, we cannot use unlimitedly large capacitors in the "DTC Bypath" design because it increases current flow and 
reduces the gain of differential mode signal voltage. For this reason, we made $C_{t 2}$ have the same order with $C_{e b}$ and swept $C_{t 1}$, which is not a perfect cancellation, but a reasonable specification to evaluate this design.

Table 3. The parameters of the simulated circuit components in Figure 3.

\begin{tabular}{cccc}
\hline Parameter & Value & Parameter & Value \\
\hline Frequency & $60 \mathrm{~Hz}$ & $V_{C M}$ & $1 \mathrm{~V}$ \\
$C_{e a}$ & $200 \mathrm{pF}$ & $C_{t 1}$ & $100 \mathrm{pF}$ to $300 \mathrm{pF}$ \\
$C_{e b}$ & $400 \mathrm{pF}$ & $C_{t 2}$ & $400 \mathrm{pF}$ \\
\hline
\end{tabular}

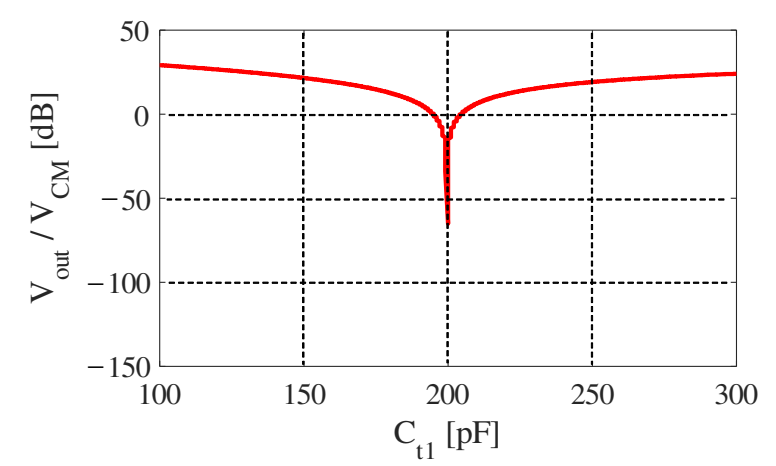

Figure 7. Simulated result of output voltage normalized to CM noise for "DTC Bypath" under Table 3's condition.

\subsection{Estimating the Practical Tunability Step of the DTCs}

In the last two sections, we used rounded values for $C_{e a}$ and $C_{e b}$. In a realistic case, these capacitances could take any value in a certain range. This may cause a gap between the simulated result and the actual performance. However, there are some facts that can help us estimate the tunability step of the DTCs, such that we can fill this gap.

The first fact is that the capacitance of a noncontact electrode generally varies in hundreds or tens of picofarad order, less than $1 \mathrm{nF}$. In this range, the two EBI capacitances are generally in the same order, such that the noise rejection is determined by the absolute value of the imbalance between them. From (3), we can also know this fact by using the actual factors: the input impedance $Z_{\text {in }}$ is high enough to eliminate the influence of $C_{e a}$ or $C_{e b}$ in the denominator, and the output voltage be determined by the absolute value of the imbalance. Because of this fact, our simulation at the rounded point can be persuasive.

The second fact is that the actual accuracy of the adjustable capacitance step can be limited by many other factors. In our idea, basically the precision of the DTC cannot be higher than picofarads order. Stray capacitances and other factors can bring varies of errors to the circuit, which is also about several picofarads. Because of the errors, it is not very practical to use large $C_{t 1}$ and $C_{t 2}$ to match (9), making the reasonable tunability step of "DTC Bypath" also stay in picofarads order.

Considering these two facts, we can know that the reasonable tunability step of the DTCs is in picofarads order.

\subsection{Frequency Characteristic}

In order to investigate the frequency characteristic of each design, small signal AC (alternating current) sweep was conducted and the results are shown in Figure 8. The frequency ranges from $0.01 \mathrm{~Hz}$ to $10 \mathrm{KHz}$. The imbalance between $C_{e a}$ and $C_{e b}$ was set to $100 \mathrm{pF}$. Parameters of the simulated circuit components are shown in Table $4 . C_{t 2}$ was fixed at a certain value and $C_{t 1}$ in "DTC Series" and "DTC Bypath" were tuned by every $10 \mathrm{pF}$ until they achieve a best case in rejecting the noise. From Figure 8, we can see that when $C_{t 1}$ is tuned, performance of both circuits get better generally, 
and "DTC Series" basically works better than "DTC Bypath" when the frequency is larger than $0.1 \mathrm{~Hz}$. Figure 8 also showed that in most frequency ranges, "DTC Bypath" could even make it worse than "NoDTC" when $C_{t 1}$ is smaller than 10.

Comparing the best cases, i.e., "DTCSeriesCt1 $=100$ " and "DTCBypathCt1 $=201$ ", $V_{\text {out }} / V_{C M}$ of "DTC Bypath" remained at a high level when the frequency was lower than $1 \mathrm{~Hz}$, while the noise reduction effect of "DTC Series" performed better than "DTC Bypath" by more than $100 \mathrm{~dB}$ at $1 \mathrm{~Hz}$. When the frequency increased, the noise reduction effect of "DTC Series" became worse. Most artifacts are at low frequency (below $100 \mathrm{~Hz}$ ). In conclusion, "DTC Series" generally works better than "DTC Bypath" in the frequency range of biopotential signals. Besides, it should be noted that the frequency response of an actual biosignal acquisition circuit can affect the result.

Table 4. Parameters of the circuit components for the alternating current (AC) sweep.

\begin{tabular}{cc}
\hline Parameter & Value \\
\hline$C_{e a}$ & $200 \mathrm{pF}$ \\
$C_{e b}$ & $100 \mathrm{pF}$ \\
$C_{t 1}$ ("DTC Series") & 50 to $100 \mathrm{pF}$ \\
$C_{t 2}$ ("DTC Series") & $200 \mathrm{pF}$ \\
$C_{t 1}$ ("DTC Bypath") & 151 to $201 \mathrm{pF}$ \\
$C_{t 2}$ ("DTC Bypath") & $100 \mathrm{pF}$ \\
\hline
\end{tabular}

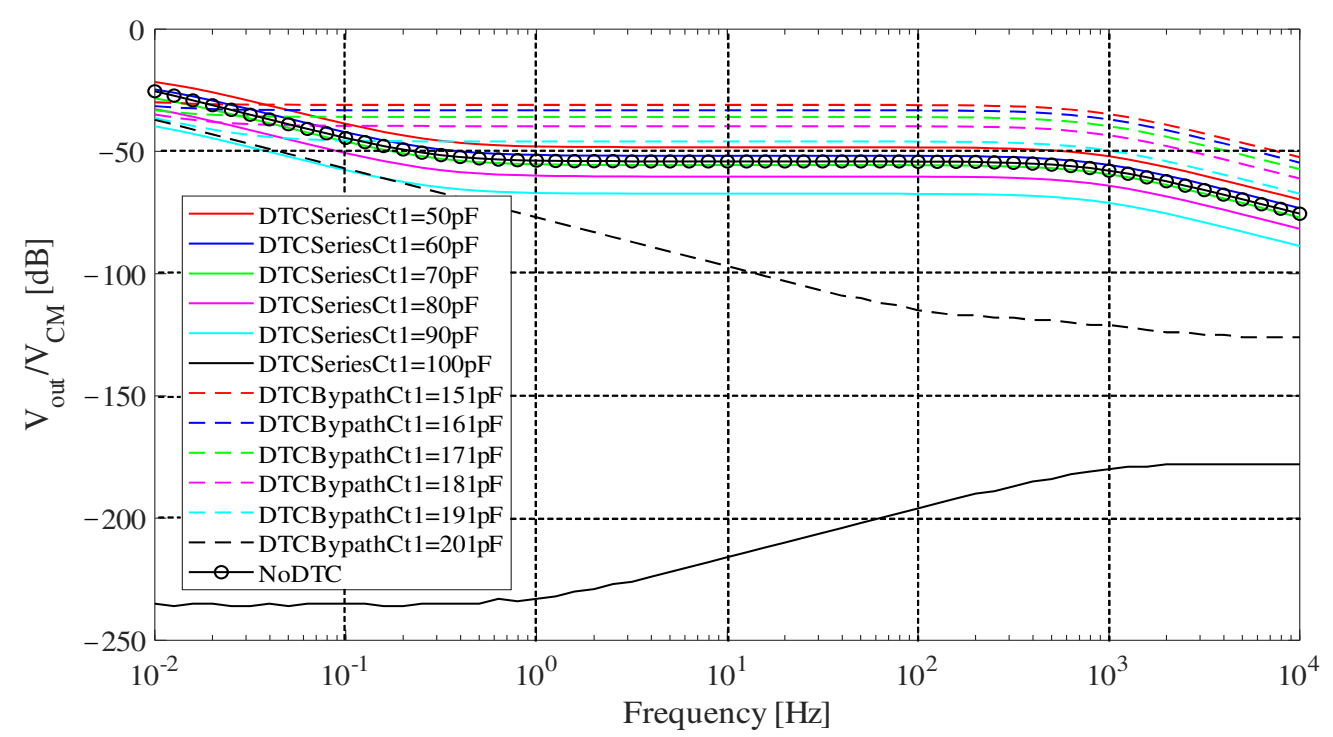

Figure 8. Simulated result of small signal AC sweep under Table 4's condition. The solid lines show results for "DTC Series", the dashed lines show results for "DTC Bypath", "NoDTC" shows the results of no imbalance cancellation.

\section{Imbalance Detection Strategy and Experimental Evaluations}

In last section, we have shown that "DTC Series" had a better performance, thus, we choose this design and perform experimental evaluations on it. In this section, we first explain our strategy to detect the imbalance of EBI impedance. After that, we present our experimental evaluation on imbalance detection and CM noise reduction effect. Earlier, we showed that if the imbalance is cancelled, the noise output of a common-mode interference voltage will be rejected to a very low level. In order to realize this goal, we must know how much imbalance exists at the EBI.

In [22], a method that uses the two voltage inputs of the differential amplifier was mentioned. However, this method has three disadvantages. The first one is that it receives many interferences. For example, when the biopotential voltage is at a high level, it may cause significant errors in deciding 
if the CM noise level is higher than the threshold or not. Our design solves this problem by employing an injection sine wave signal. The second one is that it does not have good linearity for capacitance. Figure 9 shows the difference between our approach and the conventional detection method. With good linearity, the imbalance cancellation process could be accomplished more preciously and simply.

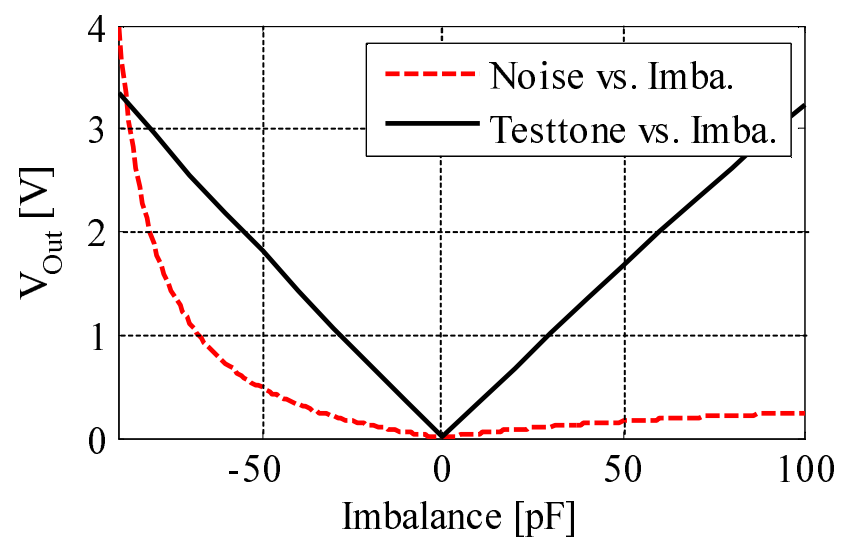

Figure 9. Correlation between the test signal and the electrode-body interface (EBI) imbalance of conventional method and our circuit design. Red dashed curve: with the conventional method (input voltage vs. the imbalance); black curve: with our design (test tone vs. the imbalance).

The third disadvantage of it is that the input voltage is difficult to filter. In the biopotential frequency range of about $0.01 \mathrm{~Hz}$ to $1 \mathrm{kHz}$, the $\mathrm{CM}$ voltage component can appear at any frequency. The conventional method cannot find out if the input voltage is really related to the CM noise source. On the other hand, our design employs a sine wave of $1 \mathrm{kHz}$, making it easy to be filtered and improve the precision of imbalance monitoring.

Figure 10 shows the configuration of our system to detect and cancel the imbalance. In order to monitor the variation of the imbalance continuously, we employed an injection signal, or what we call a "test tone" in Figure 10, which is a sine wave with a frequency of $1 \mathrm{kHz}$ and an amplitude of $100 \mathrm{mV}$. The test tone is injected at the positive input of the buffer, passing a resistor of $100 \mathrm{k} \Omega$. When the capacitance of EBI changes, output of this injection signal at $V_{\text {out }}$ follows, such that we can detect this change by reading this output. In order to figure out the exact value of the imbalance in picofarad order, we read in the amplitude and the phase information of the injection signal input and output. We can know which capacitance $\left(C_{e a}\right.$ or $\left.C_{e b}\right)$ is larger by comparing the phase, because it reverses by 180 degree depending on whether $C_{e a}$ or $C_{e b}$ is larger. Moreover, the amplitude of the output tells us the absolute value of the imbalance.

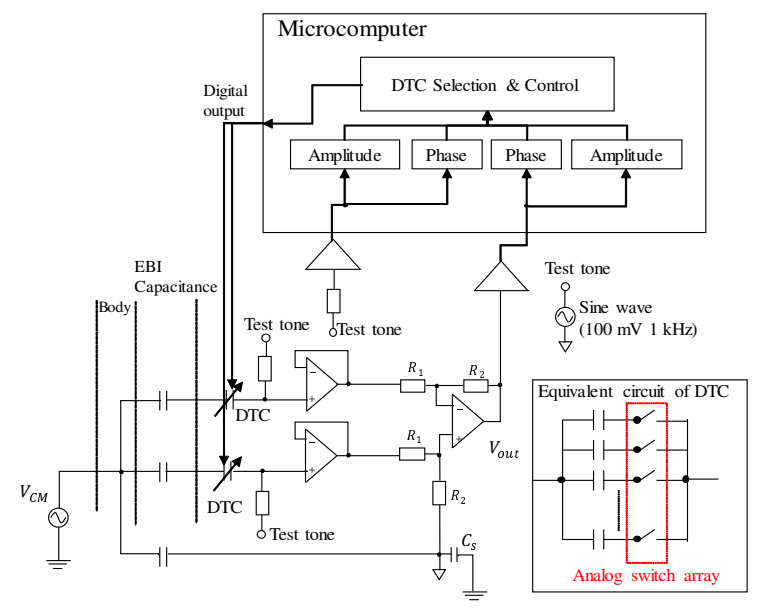

Figure 10. Configuration of the imbalance cancellation system with a microcomputer. 
Due to this, the $100 \mathrm{k} \Omega$ resistor and the capacitance of EBI and DTC forms a high-pass filter, which could cause a decline in signal gain. However, during practical use, a switch could be employed to cut off the connection at the place of $100 \mathrm{kOhms}$ resistor, such that the biosignal would only be affected during the cancellation process. When the $\mathrm{CM}$ noise level is high, we cancel the imbalance, and turn off the switch after the noise is driven down, only remaining the DTCs in the front end. Rejecting the interference of the biosignal could also be an advantage during the imbalance detection progress.

Figure 11 shows the correlation between the imbalance and the output of the injection signal. From this figure, we can see that they are almost linearly related in our experimental environments. This property can also be confirmed by circuit simulation. In Figure 10, the equivalent circuit of our originally designed DTC is also presented. It consists of several channels of capacitors connected in parallel; each channel is controlled by one analog switch. For example, in ideal case, if we make the capacitance of each channel to be $1 \mathrm{pF}, 2 \mathrm{pF}, 4 \mathrm{pF}, 8 \mathrm{pF}, 16 \mathrm{pF}, \ldots, 512 \mathrm{pF}$, then the capacitance value can be binarily tuned in the range of $1 \mathrm{pF}$ to $1 \mathrm{nF}$, by switching on or off each channel with a digital signal.

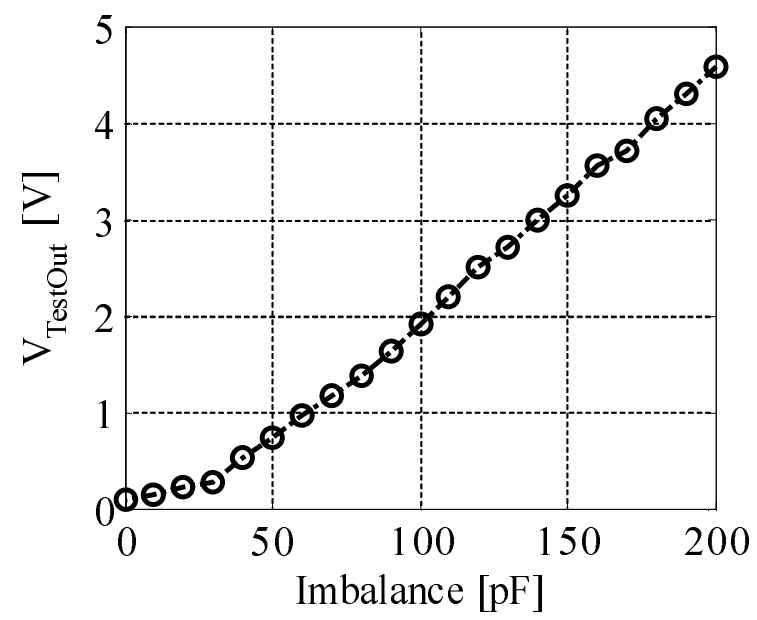

Figure 11. Correlation between the imbalance and the output of the injection signal (measured in experimental environments). When the absolute value of imbalance increases, the output voltage of test tone increases linearly.

There are some other DTCs and the equivalent circuits can be drawn. The existing three types of DTCs include the RF (radio frequency) tuning micro-electro-mechanical system (MEMS), the barium strontium titanate (BST) device and the silicon on isolator (SOI)/silicon on sapphire (SOS) tuning devices. However, none of these three types of DTC can meet our demands because all of them only work rightly in radio frequency range. Other variable capacitor elements like a varicap diode cannot be linearly tuned with a digital signal. In summary, there was no other existing DTC that can meet all our demands: availability in biopotential frequency, big tunable range (hundreds of picofarads), good linearity, and digitally tunability. Therefore, we developed our original DTC. We employed the analog switch array, or multiplexer, to construct the structure shown in Figure 10. The analog switch array that we employed consists of MOSFETs (metal-oxide-semiconductor field-effect transistor). When they work as an analog switch array, the level of gate voltage controls the current flow between source and drain, realizing a digitally controlled switch-on or -off operation. Because we connected a capacitor in series on each channel and combined all the inputs or outputs respectively, the capacitance value increases when the channel switch is on or decreases when it is off. The multiplexer reads in digital data from the microcomputer such that it can be digitally tuned.

Figure 12 shows the flow chart of our imbalance detection and DTC management algorithm. A threshold value of test tone output was used to trigger the DTC tuning operation. When the output of test tone signal increases to a high level, the microcomputer reads in the input and output of the test tone, from the two ports of our biopotential acquisition circuit shown in Figure 10. After that, values of the phase and amplitude are employed to help tuning the DTCs, as we mentioned earlier. 
Lastly, we check the output of the test tone again to confirm that the imbalance cancellation is successful. This loop is supposed to be performed in the microcomputer continuously because we expect that the CMRR can be restored automatically during actual use.

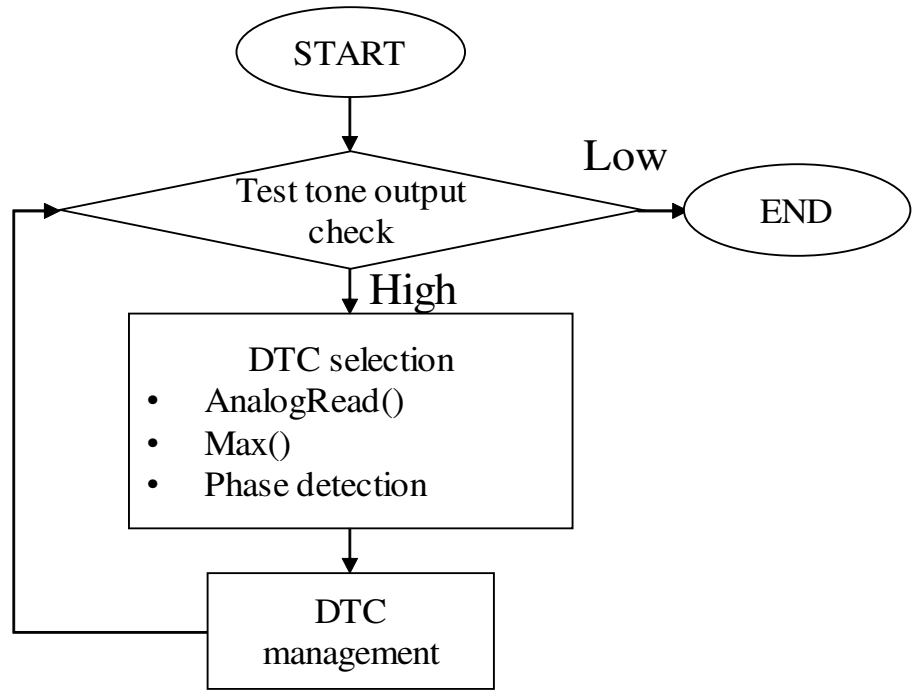

Figure 12. Flow chart of the imbalance detection and DTC management algorithm.

To make the system realize fully automatic imbalance cancellation during noncontact biopotential, acquisition is always a goal. The system and the algorithm in the microcomputer can generally work automatically, but there are still some challenges in its optimization, especially in controlling the DTCs. In this work, presenting the value and potential of the approach is our focus.

Figure 13 is a picture of our evaluation board. We constructed the system shown in Figure 10 on a universal board to experimentally evaluate the performance of our design. We use two sets of ceramic capacitors to simulate the EBI capacitance. A coupling voltage of $1 \mathrm{~V}, 60 \mathrm{~Hz}$ (also a sine wave, but different from the $100 \mathrm{mV}, 1 \mathrm{kHz}$ test tone) as a pseudo CM noise source $V_{C M}$, which was generated with a function generator. A Wien bridge oscillator circuit was developed to provide the $1 \mathrm{kHz}$ sine wave injection signal. The chip capacitors in the DTCs are soldered on the backside of this board. We first used ordinary capacitors like ceramic capacitors or mica capacitors, instead of DTCs to exactly cancel the imbalance, to see how much the CM noise is reduced when our design works with no need to worry about the digitally tuning part.

Figure 14 shows the CM noise reduction efficacy when ordinary capacitors like ceramic capacitors or mica capacitors are used to cancel the imbalance. We can see from this figure that when the imbalance is cancelled, the noise output from our evaluation board remains at a low level, around $60 \mathrm{mV}$. The noise level can be driven down by about $30 \mathrm{~dB}$ when the imbalance is as large as $200 \mathrm{pF}$. This result generally matches our simulation result, and the remaining noise voltage could be from the other parts of the circuit, like the amplifiers.

Figure 15 shows the experimental evaluation result of our originally constructed DTC. We have tried several kinds of analog switch array elements, among them ADG512 (analog devices) and MAX335EUG+ (maxim-integrated) are chosen because their features fit our demands best. Parameters of the two chips are shown in Table 5 . They can both run with $\pm 5 \mathrm{~V}$ voltage supply, and the channel number of ADG512 is 4 while MAX335EUG+ has 8 channels. The channel on-capacitance and channel off-capacitance are two important parameters, because during our test we found that they decide the equivalent capacitance of each channel. For example, the tuning step of the DTC using the ADG512 is about $24 \mathrm{pF}$, which is near to the difference between the channel on-capacitance and channel off-capacitance. The reason why it is smaller than (35 pF-24 pF) is because we connected a 
capacitor in serial with it, to make the circuit more stable. In a word, the structure of CMOS limited its tuning step range.

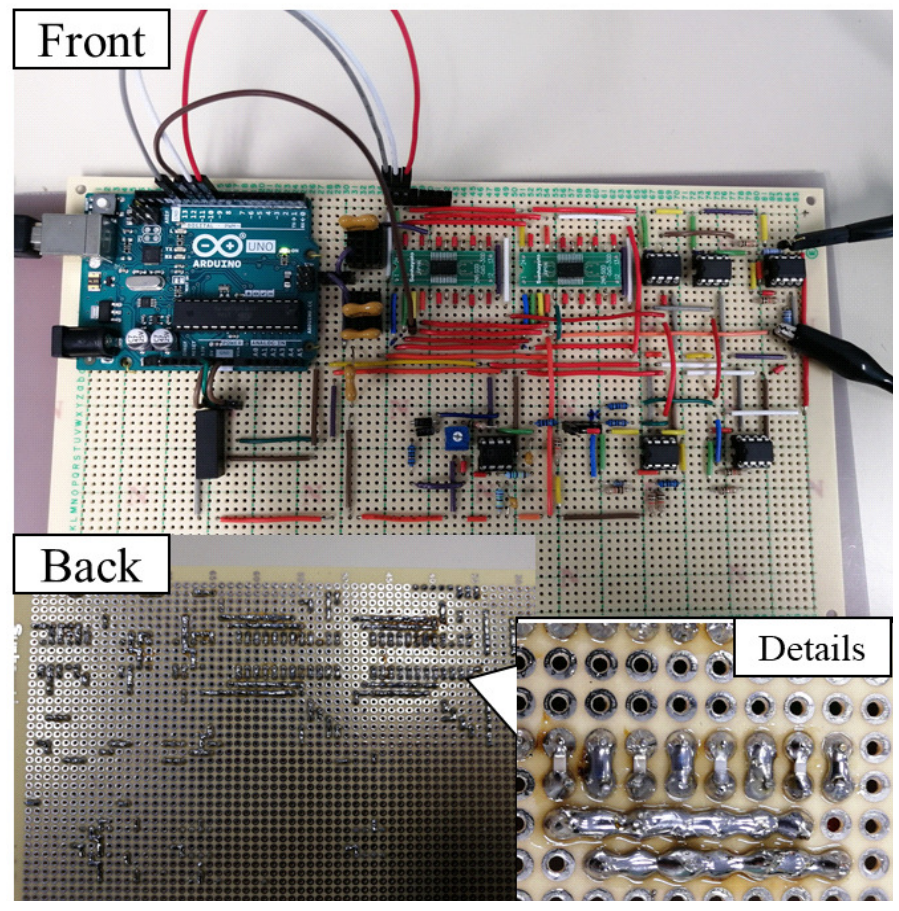

Figure 13. Evaluation circuit on a universal board. We use two sets of ceramic capacitors to simulate the EBI capacitance. The chip capacitors for constructing the DTC are soldered on the back side of the board.

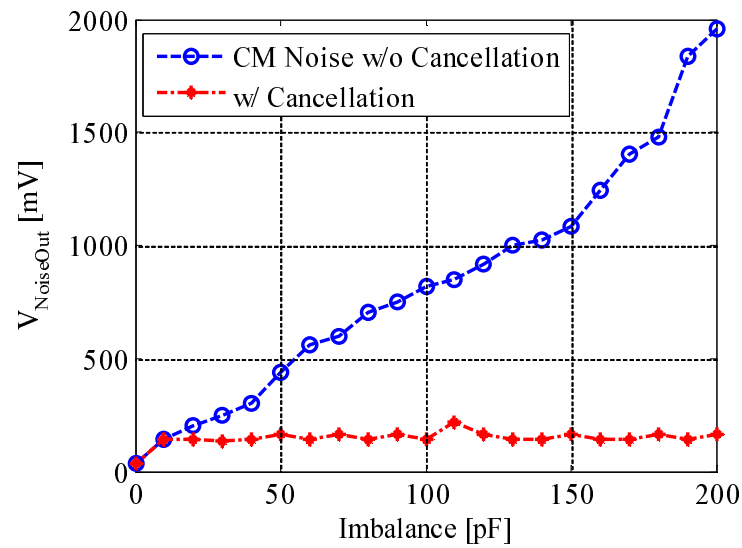

Figure 14. Noise reduction efficacy of our approach. The noise source is a $1 \mathrm{~V}, 60 \mathrm{~Hz}$ sine wave. The blue circled line is the CM noise output when the imbalance is forced in the capacitors emulating the EBIs; the red dotted line is the result when the imbalance is overcame by means of appropriate selection of ordinary capacitors (manually).

From Figure 15, we can see that the equivalent capacitance of each channel is about $24 \mathrm{pF}$ when we use the ADG512 chip. The tunable range of DTC using ADG512 is up to $96 \mathrm{pF}$, approximately. However, due to this, we used one of its channels as a switch (no capacitor connected to this channel), making the actual upper limit about $75 \mathrm{pF}$ (approximately $3 \times 24 \mathrm{pF}$ ). This value can change due to the circuit implementation and the chip capacitor we connected to it. On the other hand, MAX335EUG+ has 8 channels but the channel on-capacitance is about $8 \mathrm{pF}$. This makes the tunable range of our MAX335EUG + version of DTC only able to be tuned up to around $50 \mathrm{pF}$. Because we used one of its 
channels as a switch, the actual upper limit is about $42 \mathrm{pF}(7 \times 6 \mathrm{pF})$. According to our measurement, the equivalent capacitance of each channel is about 6 to $8 \mathrm{pF}$, which was hard to be exactly measured because it is quite a small value. Moreover, we can only give the results in tens of picofarads order in Figure 15 in the same reason.

The actual performance of our approach could still be significantly improved when better DTCs are employed in the circuit. The tuning step of the digitally tunable capacitors (DTCs) has certain influences on the accuracy of this technique. Approximately the drop of noise reduction caused by the mismatches between the EBI capacitances and the DTC can be estimated from Figure 14. For example, if the tuning step of the DTC is $24 \mathrm{pF}$, the noise output rises by about $100 \mathrm{mV}$ in the worst case.

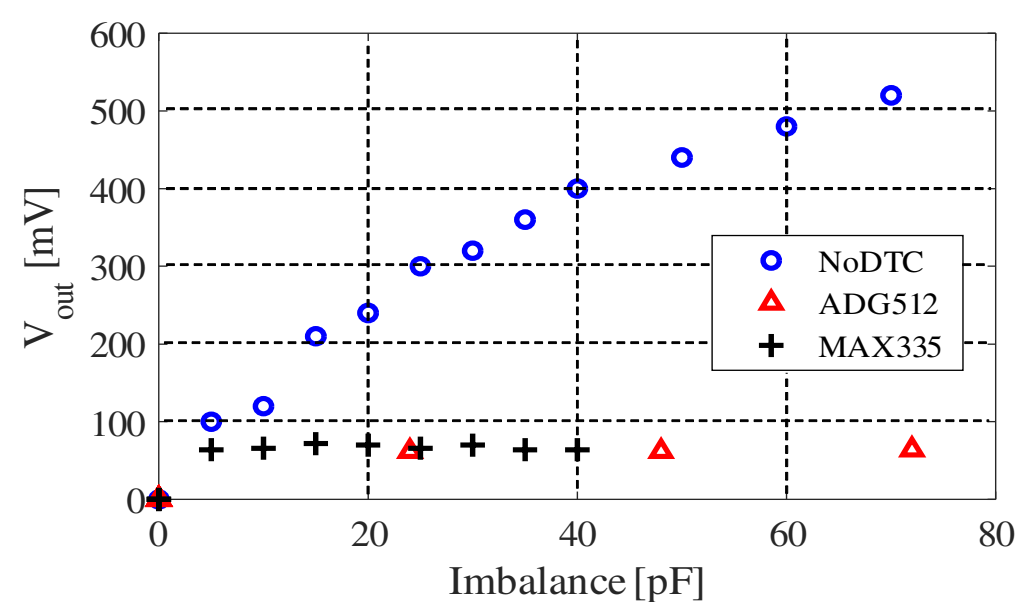

Figure 15. Experimental result of noise reduction efficacy using DTCs. ADG512: 4 tunable steps, about $24 \mathrm{pF}$ for each step; MAX335: 8 tunable steps, about 6 to $8 \mathrm{pF}$ for each step.

Table 5. Parameters of ADG512 and MAX335EUG+

\begin{tabular}{ccc}
\hline & ADG512 & MAX335EUG+ \\
\hline Voltage Supply & $\pm 5 \mathrm{~V}$ & $\pm 5 \mathrm{~V}$ \\
Number of Channels & 4 & 8 \\
Channel On-Capacitance & $35 \mathrm{pF}$ & $8 \mathrm{pF}$ \\
Channel Off-Capacitance & $9 \mathrm{pF}$ & $2 \mathrm{pF}$ \\
\hline
\end{tabular}

\section{Discussion and Summary}

Noncontact/capacitive biopotential sensing technology has a great future in medical treatment, healthcare and new applications with wearable technology. The disadvantages of the contact electrodes, about comfort and longevity, can be solved by insulated biopotential sensing technology, but the common-mode noise rejection is still a challenging task for noncontact electrodes. In this work, we have presented an approach to reduce the CM noise output of a noncontact biopotential acquisition circuit (analog front end), which works through cancelling the imbalance of EBI impedance with DTCs. At first, we provided a circuit model of noncontact biopotential acquisition, and analyzed our proposal through calculations and simulations. We clarified how much influence an imbalance in the EBI could have for a typical biopotential acquisition circuit, showing that our proposal is feasible by simulation results. We also made a comparison with another circuit design, showing the frequency sweep result of both circuits. Based on the simulation results, the first proposal that we outlined in the "DTC Series" was our last choice. Secondly, we proposed a design that uses a microcomputer together with originally designed DTC to cancel the imbalance. An injection signal was employed to detect and monitor the impedance change of the EBI, such that we can allow the microcomputer control the DTCs accordingly. The performance of this design was evaluated on a circuit board in experimental environments. When ordinary capacitors like ceramic capacitors or mica capacitors are used for evaluation, the result showed that the CM noise level can be driven down by about $30 \mathrm{~dB}$ 
when there is an imbalance of $200 \mathrm{pF}$, which matches our simulations quite well.

It is a fact that some existing tunable capacitor elements have almost the same design as our DTC. However, the novelty of our design still lies in its advantages of realizing good digital tunability and availability in our EBI imbalance cancellation circuit. In the future, we expect that new design of DTC with better linearity, larger capacitance variation and better tunability can improve the performance of this design. Embedding a tunable capacitor to make a new type of noncontact electrode is also in our vision.

Aside from this, the analog-front-end of our design have very large input impedance (more than $160 \mathrm{M} \Omega$ when OP07 is used) and low current. The main energy cost is from the microcomputer, whose power consumption can be driven down to as low as $23 \mu \mathrm{A}$ with $5 \mathrm{~V}$ power supply, in power-down sleep mode. DC power supplies of the analog elements are all provided by the microcomputer.

In summary, we presented a novel approach to reduce CM noise for noncontact biopotential acquisition, which could help support long-term, comfort and precise applications of wearable devices, human—computer interface or IoT (Internet of Things).

Author Contributions: Conceptualization, M.C., J.W. and G.F.; methodology, M.C., J.W., G.F. and J.K.; software, M.C.; validation, M.C. and J.W.; formal analysis, M.C., J.W. and D.A.; investigation, M.C., J.W. and D.A.; resources, J.W., D.A., G.F. and J.K.; data curation, M.C. and J.W.; writing-original draft preparation, M.C.; writing-review and editing, J.W. and G.F.; visualization, M.C., J.W. and J.K.; supervision, J.W., D.A. and G.F.; project administration, J.W., D.A. and J.K.; funding acquisition, J.W. and G.F. All authors have read and agreed to the published version of the manuscript.

Funding: This study was supported in part by JSPS KAKENHI Grant Number 19H02139. The authors also gratefully acknowledge financial support for this work by the Deutsche Forschungsgemeinschaft under GRK2495/A.

Conflicts of Interest: The authors declare no conflict of interest.

\section{Abbreviations}

The following abbreviations are used in this manuscript:

$\begin{array}{ll}\text { CM } & \text { Common Mode } \\ \text { CMRR } & \text { Common Mode Rejection Ratio } \\ \text { ECG } & \text { Electrocardiogram } \\ \text { EEG } & \text { Electroencephalogram } \\ \text { EOG } & \text { Electrooculogram } \\ \text { DTC } & \text { Digitally Tunable Capacitor } \\ \text { AC } & \text { Alternating Current } \\ \text { DC } & \text { Direct Current } \\ \text { OP-AMP } & \text { Operational Amplifier }\end{array}$

\section{References}

1. Astrin, A. IEEE Standard for Local and Metropolitan Area Networks_Part 15.6: Wireless Body Area Networks; IEEE: Piscataway, NJ, USA, 2012; pp. 1-271.

2. Wang, J.; Wang, Q. Body Area Communications; Wiley-IEEE: Singapore, 2013; ISBN 978-111-818-848-4.

3. Bonato, P. Wearable sensors and systems-From enabling technology to clinical applications. IEEE Eng. Med. Biol. Mag. 2010, 29, 25-36. [CrossRef]

4. Bulling, A.; Ward, J.A.; Gellersen, H.; Trösster, G. Eye movement analysis for activity recognition using electrooculography. IEEE Trans. Pattern Anal. Mach. Intell. 2011, 3, 741-753. [CrossRef] [PubMed]

5. Nopsuwanchai, R.; Noguchi, Y.; Ohsuga, M.; Kamakura, Y.; Inoue, Y. Driver-independent assessment of arousal states from video sequences based on the classification of eyeblink patterns. In Proceedings of the 11th International IEEE Conference, Intelligent Transportation Systems, 2008, ITSC 2008, Beijing, China, 12-15 October 2008; pp. 917-924. 
6. Schmidt, J.; Laarousi, R.; Stolzmann, W.; Karrer-Gauß, K. Eye blink detection for different driver states in conditionally automated driving and manual driving using EOG and a driver camera. Behav. Res. Methods 2017, 1-14. [CrossRef] [PubMed]

7. Lv, Z.; Wu, X.; Li, M.; Zhang, C. Implementation of the EOG-based human computer interface system. In Proceedings of the 2nd International Conference on Bioinformatics and Biomedical Engineering (ICBBE'08), Shanghai, China, 16-18 May 2008; pp. 2188-2191.

8. Barea, R.; Boquete, L.; Mazo, M.; Lopez, E. Wheelchair guidance strategies using EOG. J. Intell. Robot. Syst. 2002, 34, 279-299.:1016359503796. [CrossRef]

9. Venkataramanan, S.; Prabhat, P.; Choudhury, S.R.; Nemade, H.B.; Sahambi, J.S. Biomedical instrumentation based on EOG signal processing and application to a hospital alarm system. In Proceedings of the IEEE ICISIP, Chennai, India, 4-7 January 2005; pp. 535-540.

10. Richardson, P. The insulated electrode: A pasteless electrocardiographic technique. In 20th Annual Conference on Engineering in Medicine and Biology; Wellesley Press: Wellesley, MA, USA, 1967; Volume 9, pp. 15-17.

11. Wang, X.; Liu, S.; Zhu, M.; Wang, X.; Liu, Z.; Jiang, Y.; Wang, D.; Li, P.; Samuel, O.W.; Wu, W.; et al. Performance of Flexible Non-contact Electrodes in Bioelectrical Signal Measurements. In Proceedings of the 2019 IEEE International Conference on Real-Time Computing and Robotics (RCAR), Irkutsk, Russia, 4-9 August 2019; pp. 175-179.

12. Chen, M.; Chun, H.S.; Castro, I.D.; Torfs, T.; Lin, Q.; van Hoof, C.; Wang, G.; Lian, Y.; van Helleputte, N. A $400 \mathrm{G} \Omega$ Input-Impedance, 220MVpp Linear-Input-Range, 2.8Vpp CM-Interference-Tolerant Active Electrode for Non-Contact Capacitively Coupled ECG Acquisition. In Proceedings of the 2018 IEEE Symposium on VLSI Circuits, Honolulu, HI, USA, 18-22 June 2018; pp. 129-130.

13. Hsu, C.-C.; Lin, B.-S.; He, K.-Y.; Lin, B.-S. Design of a Wearable 12-Lead Noncontact Electrocardiogram Monitoring System. Sensors 2019, 19, 1509. [CrossRef] [PubMed]

14. Dabbaghian, A.; Yousefi, T.; Shafia, P.; Fatmi, S.Z.; Kassiri, H. A 9.2-Gram Fully-Flexible Wireless Dry-Electrode Headband for Non-Contact Artifact-Resilient EEG Monitoring and Programmable Diagnostics. In Proceedings of the 2019 IEEE International Symposium on Circuits and Systems (ISCAS), Sapporo, Japan, 26-29 May 2019; pp. 1-5.

15. Dong, S.; Zhang, Y.; Ma, C.; Zhu, C.; Gu, Z.; Lv, Q.; Zhang, B.; Li, C.; Ran, L. Doppler Cardiogram: A Remote Detection of Human Heart Activities. IEEE Trans. Microw. Theory Tech. 2020, 68, 1132-1141. [CrossRef]

16. Cardillo, E.; Caddemi, A. Radar range-breathing separation for the automatic detection of humans in cluttered environments. IEEE Sens. J. 2020. [CrossRef]

17. Cardillo, E.; Caddemi, A. A review on biomedical MIMO radars for vital sign detection and human localization. Electronics 2020, 9, 1497. [CrossRef]

18. Schellenberger, S.; Shi, K.; Michler, F.; Lurz, F.; Weigel, R.; Koelpin, A. Continuous In-Bed Monitoring of Vital Signs Using a Multi Radar Setup for Freely Moving Patients. Sensors 2020, 20, 5827. [CrossRef] [PubMed]

19. Michler, F.; Shi, K.; Schellenberger, S.; Lenhard, T.; Dassel, F.; Scheiner, B.; Lurz, F.; Weigel, R.; Koelpin, A. A Radar-Based Vital Sign Sensing System for In-Bed Monitoring in Clinical Applications. In Proceedings of the 2020 German Microwave Conference (GeMiC), Cottbus, Germany, 9-11 March 2020; pp. 188-191.

20. Taylor, W.; Abbasi, Q.H.; Dashtipour, K.; Ansari, S.; Shah, S.A.; Khalid, A.; Imran, M.A. A Review of the State of the Art in Non-Contact Sensing for COVID-19. Sensors 2020, 20, 5665. [CrossRef] [PubMed]

21. Ha, S.; Kim, C.; Chi, Y.M.; Akinin, A.; Maier, C.; Ueno, A.; Cauwenberghs, G. Integrated circuits and electrode interfaces for noninvasive physiological monitoring. IEEE Trans. Biomed. Eng. 2014, 61, 1522-1537. [CrossRef] [PubMed]

22. Noro, M.; Anzai, D.; Wang, J. Common-mode noise cancellation circuit for wearable ECG. Healthc. Technol. Lett. 2017, 4, 64-67. [CrossRef] [PubMed]

23. Liao, W.; Shi, J.; Wang, J. Electromagnetic interference of wireless power transfer system on wearable electrocardiogram. IET Microwaves Antennas Propag. 2017, 11, 330-335. [CrossRef]

24. Chi, Y.M.; Maier, C.; Cauwenberghs, G. Ultra-high input impedance, low noise integrated amplifier for noncontact biopotential sensing. IEEE J. Emerg. Sel. Top. Circuits Syst. 2017, 11, 330-335. [CrossRef]

25. Chen, M.; Wang, J.; Anzai, D.; Fischer, G. Common-mode noise reduction circuit design for biosignal acquisition system-In Comparison with the DRL. IEEJ Trans. Fundam. Mater. 2019, 139, 657-662. [CrossRef] 
26. Serteyn, A.; Vullings, R.; Meftah, M.; Bergmans, J.M. Motion artifacts in capacitive ECG measurements: reducing the combined effect of DC voltages and capacitance changes using an injection signal. IEEE Trans. Biomed. Eng. 2015, 62, 264-273. [CrossRef] [PubMed]

27. Degen, T.; Loeliger, T. An improved method to continuously monitor the electrode-skin impedance during bioelectric measurements. In Proceedings of the 29th Annual International Conference of the IEEE Engineering in Medicine and Biology Society, Lyon, France, 22-26 August 2007; pp. 6294-6297.

Publisher's Note: MDPI stays neutral with regard to jurisdictional claims in published maps and institutional affiliations.

(C) 2020 by the authors. Licensee MDPI, Basel, Switzerland. This article is an open access article distributed under the terms and conditions of the Creative Commons Attribution (CC BY) license (http://creativecommons.org/licenses/by/4.0/). 